\title{
A review of computer assisted detection/diagnosis (CAD) in breast thermography for breast cancer detection
}

\begin{abstract}
Breast cancer is the leading type of cancer diagnosed in women. For years human limitations in interpreting the thermograms possessed a considerable challenge, but with the introduction of computer assisted detection/diagnosis (CAD), this problem has been addressed. This review paper compares different approaches based on neural networks and fuzzy systems which have been implemented in different CAD designs. The greatest improvement in CAD systems was achieved with a combination of fuzzy logic and artificial neural networks in the form of FALCON-AART complementary learning fuzzy neural network (CLFNN). With a CAD design based on FALCON-AART, it was possible to achieve an overall accuracy of near $90 \%$. This confirms that CAD systems are indeed a valuable addition to the efforts for the diagnosis of breast cancer. Lower cost and high performance of new infrared systems combined with accurate CAD designs can promote the use of thermography in many breast cancer centres worldwide.
\end{abstract}

Keyword: Thermography; Breast cancer; Computer aided detection/diagnosis; Fuzzy logic; Neural networks 